\title{
Radiation transport modelling for the interpretation of oblique ECE measure- ments
}

\author{
Severin S. Denk ${ }^{1,2}$, Rainer Fischer ${ }^{1}$, Omar Maj ${ }^{1}$, Emanuele Poli ${ }^{1}$, Jörg K. Stober ${ }^{1}$, Ulrich Stroth ${ }^{1,2}$ \\ Vanovac $^{3}$, Wolfgang Suttrop ${ }^{1}$, Matthias Willensdorfer ${ }^{1}$, and the ASDEX Upgrade Team ${ }^{1}$ \\ ${ }^{1}$ Max Planck Institute for Plasma Physics, Boltzmannstr. 2, 85748 Garching, Germany \\ ${ }^{2}$ Physik-Department E28, Technische Universität München, 85748 Garching, Germany \\ ${ }^{3}$ FOM-Institute DIFFER, Dutch Institute for Fundamental Energy Research, $5600 \mathrm{HH}$, Eindhoven, The Netherlands
}

\begin{abstract}
The electron cyclotron emission (ECE) diagnostic provides routinely electron temperature $\left(T_{\mathrm{e}}\right)$ measurements. At ASDEX Upgrade an electron cyclotron forward model, solving the radiation transport equation for given $T_{\mathrm{e}}$ and electron density profile, is used in the framework of integrated data analysis. With this method $T_{\mathrm{e}}$ profiles can be obtained from ECE measurements even for plasmas with low optical depth. However, due to the assumption of straight lines of sight and an absorption coefficient in the quasi-perpendicular approximation this forward model is not suitable for the interpretation of measurements by ECE diagnostics with an oblique line of sight.

Since radiation transport modelling is required for the interpretation of oblique ECE diagnostics we present in this paper an extended forward model that supports oblique lines of sight. To account for the refraction of the line of sight, ray tracing in the cold plasma approximation was added to the model. Furthermore, an absorption coefficient valid for arbitrary propagation was implemented. Using the revised model it is shown that for the oblique ECE Imaging diagnostic at ASDEX Upgrade there can be a significant difference between the cold resonance position and the point from which most of the observed radiation originates.
\end{abstract}

\section{Introduction}

For the measurement of both, the electron temperature $\left(T_{\mathrm{e}}\right)$ profile and $T_{\mathrm{e}}$ fluctuations, Electron Cyclotron Emission (ECE) is a widely used diagnostic. However, every ECE diagnostic inherently measures radiation temperature $\left(T_{\text {rad }}\right)$ and not $T_{\mathrm{e}}$. For many cases it is possible to assume that fluctuations in $T_{\text {rad }}$ are identical to $T_{\mathrm{e}}$ fluctuations at cold resonance. But this procedure fails if absorption is low near the cold resonance and/or if the line of sight (LOS) is not perpendicular to the magnetic field lines. If the LOS of ECE diagnostic is not perfectly perpendicular to the magnetic field, the Doppler effect leads to a broadening of the local cyclotron frequency. If the ECE diagnostic is located on the low field side (LFS), the down-shifted emission can be reabsorbed close to the cold resonance, but the up-shifted emission cannot. Hence, the location of the measurement is not necessarily the cold resonance position. In this article an electron cyclotron forward model is introduced that allows the determination of the region in the plasma that contributes to the measured $T_{\text {rad. }}$

The article is structured as followed. In the second section that follows this introduction the forward model is introduced. Afterwards, in the third section, the limitations of the model are discussed. Finally, in the forth section. an example for the application of the forward model is given. Conclusions are drawn at the end.

\section{Electron cyclotron forward model}

The purpose of the electron cyclotron forward model is to calculate $T_{\text {rad }}$ for a given antenna pattern of the diagnostic, the $T_{\mathrm{e}}$ profile, the electron density $\left(n_{\mathrm{e}}\right)$ profile and the magnetic equilibrium. The process of calculating $T_{\text {rad }}$ can be decomposed into two steps. First ray tracing is performed to obtain the LOS of the diagnostic and, second, the radiation transport along the LOS to obtain $T_{\text {rad }}$ is calculated.

\subsection{Ray tracing in the limit of geometrical optics}

The implementation of ray tracing in the present model closely follows ref. [1]. The only distinction is that diffraction is neglected. Differently from ref [1], a single ray, rather then a whole diffracting beam, is traced, hence only standard ray tracing equations are solved [2]:

$$
\frac{\mathrm{d} \vec{x}}{\mathrm{~d} s}=\left.\frac{\partial \Lambda / \partial \vec{N}}{|\partial \Lambda / \partial \vec{N}|}\right|_{\Lambda=0} \quad \frac{\mathrm{d} \vec{N}}{\mathrm{~d} s}=-\left.\frac{\partial \Lambda / \partial \vec{x}}{|\partial \Lambda / \partial \vec{N}|}\right|_{\Lambda=0}
$$

The arclength is given by $s$ and $\vec{x}$ is the spatial coordinate. The second canocical coordinate $\vec{N}:=\frac{c_{0} \vec{k}}{\omega}$ with $\vec{k}$ the wave vector, $\omega$ the angular (measured) frequency and $c_{0}$ the vacuum speed of light. With $\Lambda$ the cold plasma dispersion relation:

$$
\Lambda(\vec{x}, \vec{N}, \omega)=|\vec{N}|^{2}-N_{\mathrm{s}, \omega}^{2}\left(X, Y, N_{\|}\right)
$$


and $N_{\mathrm{s}, \omega}$ is the cold plasma refractive index:

$$
N_{\mathrm{s}, \omega}\left(X, Y, N_{\|}\right)=1-X-X Y^{2} \frac{1+N_{\|}^{2} \pm \Delta}{2\left(1-X-Y^{2}\right)}
$$

Where "+" corresponds to the extraordinary mode (Xmode) and "-" corresponds to the ordinary mode (Omode).

$$
\Delta:=\sqrt{\left(1-N_{\|}^{2}\right)^{2}+4 N_{\|}^{2} \frac{1-X}{Y^{2}}}
$$

In equation (2) $X:=\frac{\omega_{\mathrm{p}}^{2}}{\omega^{2}}$ has been introduced with $\omega_{\mathrm{p}}=$ $\sqrt{\frac{n_{\mathrm{e}} e^{2}}{\epsilon_{0} m_{\mathrm{e}, 0}}}$ the plasma frequency and $e$ is the elementary charge. The vacuum permittivity is symbolized as $\epsilon_{0}$ and $m_{\mathrm{e}, 0}$ the electron rest mass. Furthermore, the normalized cyclotron frequency $Y:=\frac{\omega_{\mathrm{c}, 0}}{\omega}$ was introduced in equation (2), where $\omega_{\mathrm{c}, 0}=\frac{e B}{m_{\mathrm{e}, 0}}$ is the cyclotron frequency and $B$ the absolute magnetic field strength. The component of $\vec{N}$ that is aligned with the magnetic field line is denoted as $N_{\|}:=|\vec{N}| \cos \theta:=\frac{\vec{N} \cdot \vec{B}}{|\vec{B}|}$. The component perpendicular to the magnetic field lines is analogously defined to be $N_{\perp}:=|\vec{N}| \sin \theta$. For $\vec{B}$ the magnetic field ripple of ASDEX Upgrade is considered [3].

The two equations in (1) form a system of two coupled three dimensional differential equations. They are solved numerically using the DLSODE [4] algorithm. The derivatives in equation (1) are expanded using chain rules, which allows analytical evaluation of most the derivatives. Only the spatial derivatives of the numerical input quantities $\left(n_{\mathrm{e}}\right.$ profile, magnetic field and equilibrium) are performed numerically. For all interpolations the spline routines given by ref. [5] are used. The splines also provide all the required derivatives. The problem of the transition from vacuum propagation to plasma propagation is performed using root solving accounting also for the additional refraction on plasma entry via Snell's law. The first point of plasma propagation is given by the outermost point given by the input density profile.

To assure that the implementation of ray tracing is correct, the present model was benchmarked against the TORBEAM code [6]. Although TORBEAM does account for diffraction, the central ray of a TORBEAM calculation is only subject to refraction. In the following, one of the benchmarking scenarios is discussed.

For this particular case the LOS is reflected near the plasma core. As an example a channel of the slightly oblique ECE Imaging system (ECEI) array [7] at ASDEX Upgrade was chosen (s. a. section 4). As plasma scenario \#30839 at $t=1.75 \mathrm{~s}$ was selected. In the top graph of figure 1 the poloidal cross section of ASDEX Upgrade is shown. Flux surfaces are depicted as black dashed lines and the separatrix is highlighted with a blue solid line. In the bottom of figure 1 the top view of ASDEX Upgrade is shown. In both graphs the LOS according to the presented model (green) and TORBEAM [6] (dashed purple) are shown. On the entire LOS up shortly before the reflection the two rays do not deviate by more than $2 \mathrm{~mm}$. This deviation is expected when comparing ray tracing codes,
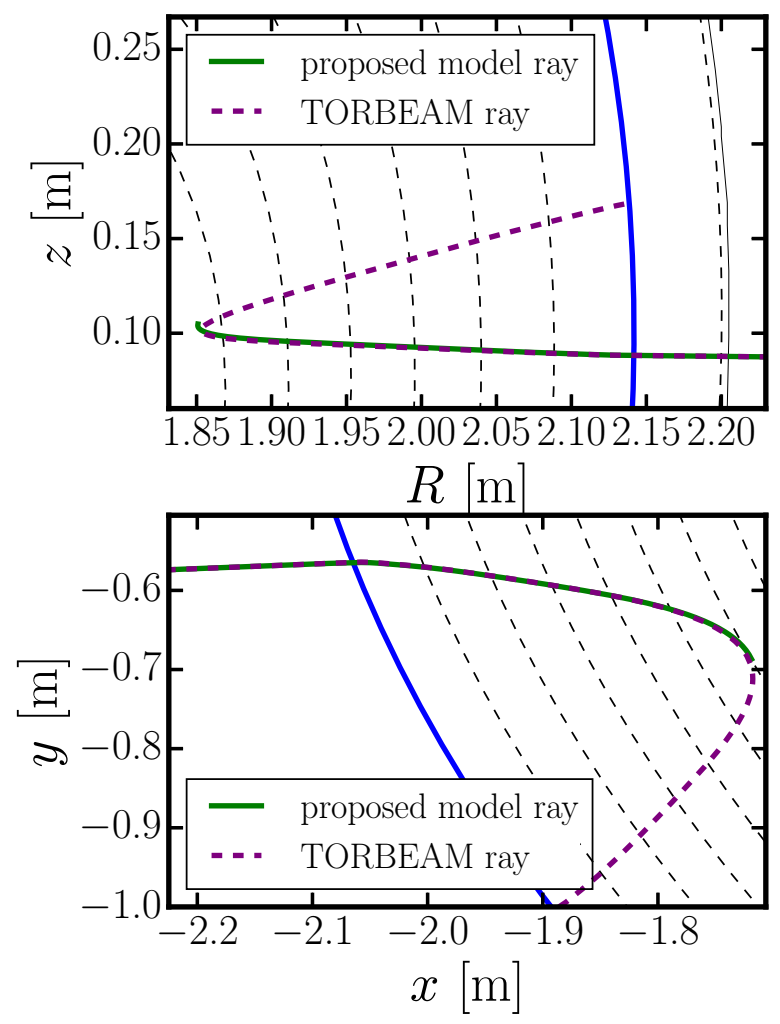

Figure 1. The top graph shows the poloidal cross section of ASDEX Upgrade for \# 30839 at $t=1.75 \mathrm{~s}$. The bottom graph shows the corresponding top view. Both graphs show one of the LOSs of the ASDEX Upgrade ECEI system. The green LOS is obtained by the present model and the purple LOS was calculated by the TORBEAM code (s. ref. [6]).

especially if refraction is strong (s. a. ref. [8]). But even this small deviation has a strong effect on the reflection, showing how strongly the reflection is altered by slight changes in the ray properties. For this reason propagation is stopped in the present model upon a sign change in $\frac{\partial|\vec{b}|}{\partial s}$. Therefore, multiple passes through a resonant layer by an internal reflection are explicitly excluded from the model. A direct consequence of this is that an internal reflection by the plasma is treated like a wall reflection with the corresponding inaccuracies. A description of the model for wall reflections can be found in section 2.3. In section 3.1 it is discussed, how reflections (both by the wall or by the plasma) affect the accuracy of the model.

\subsection{Radiation transport and EC absorption}

In this section the modelling of the radiation transport is explained. Only a single ray and measured frequency are considered. Hence, for each measured frequency the radiation transport equation has to be solved only once. The radiation transport equation (5) is given as [9]:

$$
\frac{\mathrm{d}}{\mathrm{ds}} \frac{I_{\omega}(s)}{N_{\omega, \text { ray }}^{2}(s)}=\frac{1}{N_{\omega, \text { ray }}^{2}(s)}\left(j_{\omega}(s)-\alpha_{\omega}(s) I_{\omega}(s)\right)
$$


Integrating equation (5) over the LOS yields the intensity $I_{\omega}\left(s_{\text {ant }}\right)$ at the antenna position $s_{\text {ant }}$. Furthermore, $s$ is the LOS coordinate, $N_{\omega, \text { ray }}$ the ray refractive index , $j_{\omega}(s)$ the emissivity and $\alpha_{\omega}(s)$, where introduced in equation (5). Although the "ray refractive index" $N_{\omega, \text { ray }}$ appears in the radiation transport equation it can be eliminated with a simple substitution $\frac{I_{\omega}(s)}{N_{\omega, \text { ray }}^{2}(s)} \rightarrow \Xi_{\omega}(s)$ :

$$
\frac{\mathrm{d}}{\mathrm{ds}} \Xi_{\omega}(s)=\frac{j_{\omega}(s)}{N_{\omega, \text { ray }}^{2}(s)}-\alpha_{\omega}(s) \Xi_{\omega}(s)
$$

Solving equation (6) yields $\Xi_{\omega}\left(s_{\text {ant }}\right)$. At the antenna position, however, there is no plasma and $N_{\omega, \text { ray }}^{2}\left(s_{\text {ant }}\right)=1$ and $\Xi_{\omega}\left(s_{\text {ant }}\right)=I_{\omega}\left(s_{\text {ant }}\right)$. Hence, $N_{\omega, \text { ray }}^{2}(s)$ is not needed in the radiation transport equation as long as $\frac{j_{\omega}(s)}{N_{\omega, \text { ray }}^{2}(s)}$ is used in place of $j_{\omega}(s)$. The radiation transport equation can be solved by a conventional, explicit Runge-Kutta scheme (e.g. Rk4). Both, the absorption coefficient and the emissivity are strongly peaked near the cold resonance position. Hence, it is useful to choose a smaller step size close to the cold resonance [10].

To solve the radiation transport equation both, the emissivity $j_{\omega}(s)$ and the absorption coefficient $\alpha_{\omega}(s)$, are required. In the present model the electrons are assumed to be in thermal equilibrium. This allows the usage of Kirchhoff's law to calculate the emissivity from the absorption coefficient:

$$
j_{\omega, n}(s)=\alpha_{\omega}(s) I_{\mathrm{BB}, \omega}(s) \approx \alpha_{\omega}(s) N_{\omega, \text { ray }}^{2} \frac{\omega^{2} T_{\mathrm{e}} k_{\mathrm{b}}}{8 \pi^{3} c_{0}^{2}}
$$

$I_{\mathrm{BB}, \omega}$ is the black body intensity. Applying the RayleighJeans approximation yields the expression given on the right side of equation (7) for $I_{\mathrm{BB}, \omega}$ [11]. Equation (7) can be rearranged to deliver $\frac{j_{\omega, n}(s)}{N_{\omega, \text { ray }}^{2}}$. Hence, it is enough to compute the ratio $\frac{j_{\omega, n}(s)}{N_{\omega, \text { ray }}^{2}}$ and this is possible without explicitly evaluating $N_{\omega, \text { ray }}^{2}$, cf. equation (6).

For the absorption coefficient an almost entirely analytic formulation is used, which is given by ref. [12]. The advantage of this method is given by very high numerical stability and the low requirement in computational effort. Such advantages are desirable, if the forward model is to be used in a gradient based optimization framework like IDA (s. a. ref. [10]). For a moderately dense plasma with large $T_{\mathrm{e}}$ the absorption coefficient of the $n$-th harmonic can be approximated by (s. ref. [12], specifically equation (2a) and (10a)):

$$
\begin{aligned}
\alpha_{\omega, \mathrm{n}}(s) \approx & 4 \pi^{2} \frac{\omega_{\mathrm{p}}^{2} \mu}{c_{0} \omega} \iint \mathrm{d} u_{\perp} \mathrm{d} u_{\|} \frac{u_{\perp}}{\gamma} \times \\
& \underbrace{\left(\frac{n}{\bar{\omega} N_{\perp}}\right)^{2}\left|\left(e_{x}+\frac{\bar{\omega} N_{\perp}}{n} u_{\|} e_{z}\right) J_{n}(b)-\frac{i b}{n} J_{n}^{\prime}(b) e_{y}\right|^{2}}_{\text {"Polarization factor" }} \times \\
& \underbrace{f_{\mathrm{MJ}}\left(s, u_{\perp}, u_{\|}\right)}_{\mathrm{EMD}} \underbrace{\delta\left(\gamma-u_{\|} N_{\|}-\frac{n}{\bar{\omega}}\right)}_{\text {Resonance cond. }}
\end{aligned}
$$

Equation (8) contains an integral in cylindrical, dimensionless momentum space. The symbols $u_{\perp / \|}=$ $p_{\perp / \|} /\left(c_{0} m_{\mathrm{e}, 0}\right)$ corresponds to the (dimensionless) momentum perpendicular/parallel to the magnetic field. The Lorentz factor $\gamma$ is given by $\gamma=\sqrt{1+u_{\perp}^{2}+u_{\|}^{2}}$. The inverse normalized cyclotron frequency is given by $\bar{\omega}:=\frac{\omega}{\omega_{\mathrm{c}, 0}}$ and $\mu:=\frac{m_{\mathrm{e}, 0} c_{0}^{2}}{T_{\mathrm{e}}}$. The $\delta(\ldots)$ in equation (8) represents a Dirac delta function. The $J_{n}(b)$ stands for the Bessel function of first kind and $n$-th order and $b:=\bar{\omega} N_{\perp} u_{\perp}$. Finally, $e_{x}, e_{y}, e_{z}$ are the components of the polarization vector. The mode selection is performed by the choice of $N_{\omega}$ and the polarization vector. Note that most of these quantities carry an implicit dependence on LOS position $s$. To allow for arbitrary propagation angles, the quasiperpendicular approximation (assuming $N_{\|}<v_{\mathrm{t}} / c_{0}$ with $v_{\mathrm{t}}$ the thermal electron velocity) that is utilized in ref. [12] is not applied in the implementation. In the forward model equation (8a) of reference [12] is implemented for the calculation of the absorption coefficient. In this equation the individual terms that build up the absorption coefficient are not as clearly visible as in equation (8), but still there are several simplifications that are advantageous for the implementation. The most notably distinction between equation (8) and equation (8a) of reference [12] is that the latter holds the solution of the integral over $u_{\perp}$ in equation (8). The remaining integration over $u_{\|}$is performed numerically in the model (Gaussian quadrature). To obtain $\alpha_{\omega}(s)$ the contribution of all individual harmonics $n=1, \ldots, \infty$ has to be summed up. For ASDEX Upgrade typical $T_{\mathrm{e}}$ and for the various ECE viewing geometries available at ASDEX Upgrade [7, 10] it is generally only necessary to consider the contribution of harmonics that have a cold resonance in the plasma. For X-mode ECE diagnostics located on the LFS, the first harmonic is also irrelevant, since the resonance lies in cut-off.

The contributions to the absorption coefficient can be split into three components as shown in equation (8), i.e. the "polarization factor" [12], the electron momentum distribution (EMD) and (relativistic) resonance condition. For the EMD a relativistic Maxwellian is used also known as Maxwell-Juettner distribution. For the meaning and implications of the resonance conditions we refer to ref. [11]. The "polarization factor" accounts not only for wave polarization, but also for finite Larmor radius effects [12]. For the wave polarisation the cold plasma approximation is used in ref. [12] and this is also implemented in the present model. For further details we refer to [2, 12].

\subsection{Wall reflections}

At frequencies for which the cold resonance lies near the plasma edge or in the scrape-off layer (SOL), the optical depth $\left(\tau_{\omega}\right)$ is small. Therefore, it is possible that the plasma is not optically thick (i.e. $\tau_{\omega}<3$ [2]) after a single pass through the plasma. Since the reflectivity of the wall material is hight the LOS is reflected on the high field side (HFS) and passes through the plasma a second time. Depending on the optical depth of the plasma this process can occur consecutively multiple times, which increases the total length of the LOS and if the reflect LOS passes 
through resonant layers also the observed intensity. To account for this process, a model of infinite reflections is employed using the wall reflection coefficient $R_{\text {wall }}$ as a (free) parameter. Since all ECE diagnostics at ASDEX Upgrade have a slight toroidal inclination the ray does not pass through the LFS port when reflected back from the HFS. Instead the ray hits again a wall tile and is reflected back towards the HFS. This motivates to approximate the vessel wall as two plain mirrors facing each other. Assuming that the radiation of the plasma $I_{\omega}$ and the reflectivity of the wall $R_{\text {wall }}$ is constant for each reflection, the relation between observed intensity and the intensity of a single pass is given by [13]:

$$
\kappa_{\mathrm{Refl}}:=\frac{I_{\omega, \mathrm{Refl}}}{I_{\omega}}=\frac{1}{1-R_{\mathrm{wall}} e^{-\tau_{\omega}}}
$$

At low optical depth $\kappa_{\text {Refl }}$ can be larger than two, meaning that the lengthening of the LOS due to wall reflections is a dominant effect. The consequences of this for the validity range of the model is discussed in section 2.3.

\subsection{The birthplace distribution of observed intensity}

To illustrate which portion of the plasma contributes to the measured intensity, a birthplace distribution of observed intensity (BPD) $D_{\omega}(s)$ can be defined:

$$
D_{\omega}(s):=\frac{j_{\omega}(s) T_{\omega}(s)}{I_{\omega}\left(s_{\mathrm{ow}}\right)}
$$

In equation (10) the the transmittance $T_{\omega}(s)$ of the plasma for waves at frequency $\omega$ along the LOS from point $s$ to the observation point $s_{\text {ow }}$ was introduced:

$$
T_{\omega}(s):=\mathrm{e}^{-\tau_{\omega}(s)}=\exp \left(-\int_{s}^{s_{\mathrm{ow}}} \alpha_{\omega}\left(s^{\prime}\right) \mathrm{d} s^{\prime}\right)
$$

A function similar to equation (10) is frequently used in the literature (e.g. [14], [15]), but never in conjunction with a proper normalization. To obtain the BPD the integral representation [16] of the radiative transport equation (5) is required, where the boundaries are given by the start and end points of the LOS (e. g. inner wall $s_{\text {iw }}$ and outer wall $s_{\mathrm{ow}}$, if there is no internal reflection):

$$
I_{\omega}\left(s_{\mathrm{ow}}\right)=\int_{s_{\mathrm{iw}}}^{s_{\mathrm{ow}}} j_{\omega}(s) T_{\omega}(s) \mathrm{d} s
$$

Normalizing the argument of the integral in equation (12) to the observed intensity $I_{\omega}\left(s_{\text {ow }}\right)$ yields the BPD (see equation (10)). How useful the BPD is, is demonstrated using discharge \# 30839 at $t=1.75 \mathrm{~s}$ as an example. The same channel of the slightly oblique ECE Imaging diagnostic at ASDEX Upgrade is picked as for the benchmark of the ray tracing. In figure 2 the top shows both the emissivity $j_{\omega}$ (blue line) and the transmittance $T_{\omega}$ (black dashed line) as a function of LOS coordinate $s$. At the bottom the BPD (red line) is shown as well as $T_{\mathrm{e}}$ (dotted black line). One can see that although the emissivity is non-zero for a wide region, the BPD remains zero due to the extremely

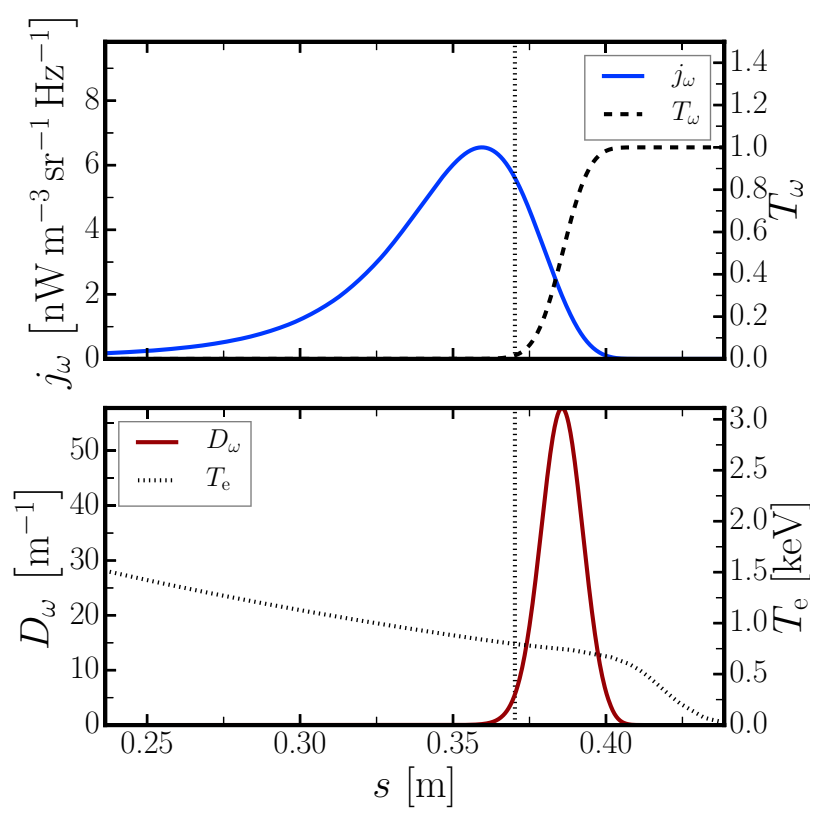

Figure 2. Emissivity, transmittance (top), BPD and $T_{\mathrm{e}}$ (bottom) for \# 30839 at $t=1.75 \mathrm{~s}$. The corresponding LOS is shown in figure 1 .

small transmittance. Even near the cold resonance position (vertical dashed line) the transmittance is still almost zero due to an optical thick layer shortly after the cold resonance. This layer is formed by the electrons, which have their cyclotron frequency up-shifted by the Doppler-shift. In this layer the transmittance then rises to one and the BPD becomes non zero. One can then use the maximum of the BPD as a corrected resonance position or "warm" resonance position [14]. Alternatively one can use the first moment of the BPD as a weighted center of the emission line [17].

\section{Limitations of the presented model}

The model presented in the previous section has two major limitations. The strongest is the influence of wall reflections or multiple passes through a resonance at extremely low optical depths. The second, weaker limitation are plasmas, where $\omega_{\mathrm{c}} \approx \omega_{\mathrm{p}}$. This section delivers a brief discussion about the validity range of the model.

\subsection{Minimum optical depth for accurate modelling}

At low enough optical depth wall reflections can enhance the observed $T_{\text {rad }}$ by an order of magnitude. Unfortunately accounting for the chaotic ray dynamics originating from multiple reflections of rays on the complex structures of the vessel is computationally far too expensive to be used in forward modelling. Empirically it was observed, however, that the simple model of two plain mirrors facing each other can deliver good results. Using the simplified reflection model presented in section 2.3 for the profile radiometer at ASDEX Upgrade the ECE measurements 
could be described up to low optical depth [10]. However, the profile radiometer at ASDEX Upgrade is situated in the mid plane. This means that the poloidal inclination of the LOS is only few degrees. Hence, the model of the two mirror represents the situation of the LOS being reflected on the torus wall adequately. For larger poloidal inclinations of the LOS the cold resonance position changes after each reflection since the LOS does not hit the wall perpendicularly. Also in the case of an internal reflections, it is usually the case that the second pass does not have the same cold resonance as the first. Therefore, the assumption that each reflection contributes as much radiation as the first pass is poor. For this reason the impact of wall reflections onto the forward modelled $T_{\text {rad }}$ is studied. Given an uncertainty of the measured $T_{\text {rad }}$ of $10 \%, \kappa_{\text {Refl }}<1.1$ for wall reflections to affect the measured $T_{\text {rad }}$ also by $10 \%$. Using equation (9) and a reasonable $R_{\text {wall }} \approx 0.9$ [10] this means that for $\tau_{\omega}>2.3$ wall reflections increase the measured $T_{\text {rad }}$ by $10 \%$.

\subsection{Limitiations of the analytic absorption formula}

The absorption coefficient presented in ref. [12] is optimized to accurately predict the microwave radiation losses through high harmonics in high $T_{\mathrm{e}}$ plasmas. Therefore, the applicability of equation (8) for ECE forward modelling of low needs to be investigated. In the following the approximations used in ref. [12] are reviewed and their effect onto the validity range of the present model are discussed.

In the formulation presented in ref. [12] there are two major approximations that have consequences for the model. The first is that only the electromagnetic component of the energy flux density is considered [12, 15, 18]. The second is that both refractive index and wave polarization are considered in the cold plasma limit. This renders the absorption coefficient to be not applicable at all for the first harmonic $[2,18]$. For harmonics with $n>4$ the harmonic overlap does not allow the expansion into single harmonics [2]. For the regime, where harmonics start to overlap, the sum over infinite harmonics needs to be carried out asymptotically. An analytical formula for this sum can be found in ref. [12]. However, for the application at ASDEX Upgrade the main interest is in the emission of the second harmonic X-mode.

For the second harmonic, strictly speaking, the accuracy of the absorption coefficient is only guaranteed, if $[2,12,18]$ :

$$
\omega_{\mathrm{c}, 0}^{2} \gg \omega_{\mathrm{p}}^{2}
$$

Since the main target of the model is to predict $T_{\text {rad }}$ for ECE measurements around the second harmonic X-mode, the applicability of the absorption coefficient given by [12] needs to be discussed in further detail.

Generally the condition given by expression (13) is only met at ASDEX Upgrade for low density plasmas. However, the sensitivity of $T_{\text {rad }}$ on the magnitude of the absorption coefficient is low, as long as the optical depth $\tau_{\omega}>3$. Only at ECE measured frequencies corresponding to the plasma edge and SOL, where the optical depth is low, $T_{\text {rad }}$ becomes dependent on the absolute magnitude of the absorption coefficient. To asses the sensitivity a benchmark was performed using the ASDEX Upgrade profile radiometer antenna pattern (s. [10] for details about this diagnostics). For a wide range of $T_{\mathrm{e}}$ and $n_{\mathrm{e}}$ profiles $T_{\mathrm{rad}}$ was then calculated using the model presented in section 2 . For the comparison the same scenarios were also modelled using the absorption coefficient derived from the fully relativistic dispersion relation as described by ref. [19]. The results are the following. In general the absorption coefficient used in the present model approximates the fully relativistic absorption coefficient adequately, even if condition given by expression (13) is violated. In most cases the relative deviations between the two absorption coefficients is smaller than $20 \%$. Even if this threshold is exceeded, the effect on $T_{\text {rad }}$ remains small as the absolute value of the absorption coefficient influences the BPD only weakly. However, there is the exception of a resonance coinciding with a reflection. Close to a reflection equation (8) becomes inaccurate, since the condition expressed by equation (13) is strongly violated. Furthermore. the ray tracing is inaccurate due to the omission of relativistic effects. In all other cases the "warm resonances" obtained with both the absorption coefficient presented in section 2 and the one given in ref. [19] deviate only by less than $3 \mathrm{~mm}$ as is demonstrated with an example in the following section.

\section{Example: Birthplace distribution of observed intensity for the slightly oblique ECE Imaging diagnostic at ASDEX Upgrade}

The ECEI at ASDEX Upgrade [7] consists of two separate imaging diagnostics. Through the usage of two opposite signed toroidal angles the two arrays allow measurements in $3 \mathrm{D}$. Although the toroidal deviation from the perpendicular is small (e.g. about $7^{\circ}$ at launch for \#30839), the Doppler-Effect has significant influence onto the center of the BPD. This is demonstrated using the following example. In discharge \# 30839 the ECEI system was used to measure the plasma displacement by magnetic perturbation coils [20]. For this purpose the ECEI observed the plasma edge near the separatrix. As described in ref. [20] the measurement position is not adequately captured by the cold resonances. However, through the usage of the model described in section 2 it was possible to recover the actual measurement positions using the maximum of the BPD as "warm" resonance position.

In figure 3 the expected measurement positions of the first ECEI array are shown in the cross section of ASDEX Upgrade for discharge \#30839 at $t=1.75 \mathrm{~s}$. All channels for which the measured optical depth $\tau<0.05$ were removed from the plot, since they lie far outside the validity regime of the model. If one assumes that the measurement positions are well described by the cold resonances (red stars) then, as shown in figure 3 , one would expect the array to cover a large portion of the plasma edge and 


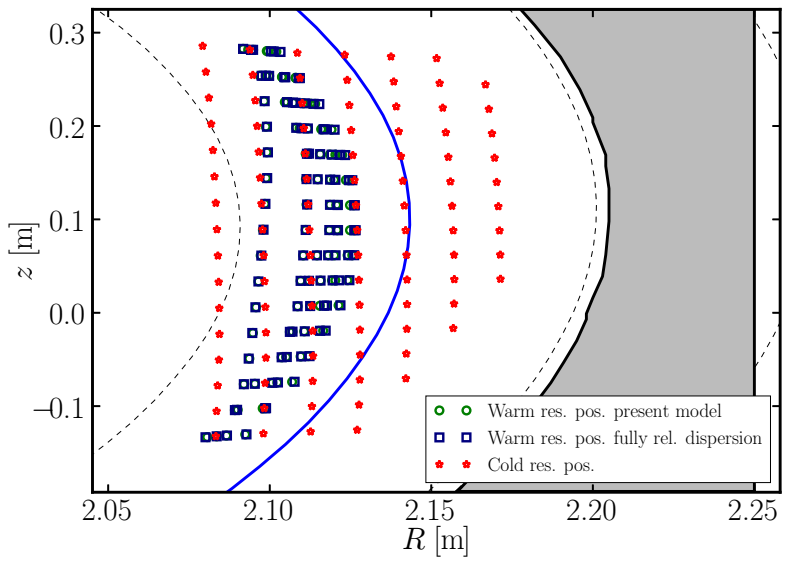

Figure 3. Poloidal cross section of ASDEX Upgrade for discharge \# 30839 at $t=1.75 \mathrm{~s}$. The dashed black lines show flux surfaces and the blue line marks the separatrix. The grey structure outlined by a solid black line depicts a piece of the outer vessel wall.

the SOL. But, if radiation transport is considered, the expected measurement position shrink together to a thin band shortly inside the separatrix (green circles). For the channels in the SOL this is due to the shine-through originating from the pedestal top. For the channels further inside the plasma the "warm" resonances are shifted outward by the Doppler effect. In figure 3 a third set of warm resonance positions, marked by blue squares, is shown. These warm resonances are obtained if the absorption coefficient discussed in section 2 is replaced by the absorption coefficient obtained from the fully relativistic dispersion relation (s. ref [19]). The "warm" resonance positions according to the absorption coefficient presented in section 2 and the absorption coefficient obtained from the fully relativistic dispersion relation are displaced at their maximum by $2 \mathrm{~mm}$. This is much smaller than the accuracy of the measurements used for the prediction of the model. Hence, the absorption coefficient presented in section 2 is at least sufficient in this case for the purposes of determining warm resonances for oblique ECE diagnostics like the ECEI diagnostic at ASDEX Upgrade.

\section{Conclusions}

A model for the radiation transport consideration refraction in the cold plasma limit has been presented. This model makes use of the approximation for the absorption coefficient presented in ref. [12]. The limitations of the model due wall reflections and internal reflections at low optical depth have been discussed. Furthermore it was shown that for the slightly oblique ECEI at ASDEX Upgrade the "warm" resonances obtained can differ significantly from the cold resonances. Lastly it was demonstrated, that the approximation used for the absorption coefficient is adequate for the calculation of warm resonances for oblique ECE diagnostics.

\section{Acknowledgements}

The author acknowledges Daniela Farina and Lorenzo Figini for providing a code for the calculation of the absorption coefficient from the fully relativistic dispersion relation.

This work has been carried out within the framework of the EUROfusion Consortium and has received funding from the Euratom research and training programme 2014-2018 under grant agreement No 633053. The views and opinions expressed herein do not necessarily reflect those of the European Commission.

\section{References}

[1] D. Farina, FUSION SCI TECHNOL 52, 154 (2007)

[2] M. Bornatici et al., Nuclear Fusion 23, 1153 (1983)

[3] R. Dux et al., Tungsten as first wall material in ASDEX Upgrade, in 21st IAEA Fusion Energy Conference (International Atomic Energy Agency, 2007)

[4] A.C. Hindmarsh, Scientific Computing 1, 55 (1983)

[5] P. Dierckx, Curve and surface fitting with splines (Oxford University Press, 1995)

[6] E. Poli et al., Comput. Phys. Commun. 136, 90 (2001)

[7] I.G.J. Classen et al., Rev. Sci. Instrum 85, (2014)

[8] R. Prater et al., Nuclear Fusion 48, 035006 (2008)

[9] G. Bekefi, Radiation processes in plasmas, Wiley series in plasma physics (Wiley, 1966)

[10] S.K. Rathgeber et al., PPCF 55, 025004 (2013)

[11] I. Hutchinson, Principles of Plasma Diagnostics (Cambridge University Press, 1987)

[12] F. Albajar et al., PPCF 49, 15 (2007)

[13] N. Luhmann Jr et al., Fusion Sci. Technol. 53, 335 (2008)

[14] G. Garstka, M. Austin, R. Ellis, Fusion Engineering and Design 53, 123 (2001)

[15] A.I. Smirnov et al., JETP 83, 294 (1996)

[16] L. Yong et al., Chinese Physics B 21, 045201 (2012)

[17] N.B. Marushchenko et al., FUSION SCI TECHNOL 50, 395 (2006)

[18] M. Bornatici et al., PHYS PLASMAS 1, 189 (1994)

[19] D. Farina, FUSION SCI TECHNOL 53, 130 (2008)

[20] M. Willensdorfer et al., submitted to PPCF (2016) 